\section{Variation of Species.}

ON p. I8I of Wallace's "Darwinism," ed. I889, this passage occurs:-" Let us suppose that a given species consists of IOO,OOO individuals of each sex, with only the usual amount of fluctuating external variability. Let a physiological variation arise, so that Io per cent. of the whole number-10,000 individuals of each sex-while remaining fertile inter se become quite sterile with the remaining 90,000 . This peculiarity is not correlated with any external differences of form or colour, or with inherent peculiarities of likes or dislikes leading to any choice as to the pairing of the two sets of individuals. We have now to inquire, What would be the result?"

I have here attempted to investigate this question algebraically.

A. We shall suppose, as Dr. Wallace does, that the number of males in the species is the same as the number of females. Each of these numbers we shall denote by unity. For convenience, we shall speak of the number of either sex as the number of the species. Let then

and

$$
x=\text { the number of the normal species, }
$$

$$
y=\text { the number of the variant variety, }
$$

at any given stage of the change above described.

If in any given generation $(x, y)$, the ratio, which the number of the variant individuals, born in a family of the normal species, bears to the total number of young born in that family, be denoted by $k$; and if $\left(x^{\prime}, y^{\prime}\right)$ denote the generation which succeeds $(x, y)$; then must

with the relation

$$
x^{\prime}: y^{\prime}:: x^{2}(1-k): y^{2}+k x^{2}
$$

$$
x^{\prime}+y^{\prime}=x+y=1 ;
$$

because the total species remains constant in number of individuals.

Now, if the variants succeed in establishing themselves as a new species, and the above two generations belong to the permanently settled state of the whole species, we must have $x^{\prime}=x$, and $y^{\prime}=y$.

Consequently, to determine $x, y$ under this condition we have the equations

$$
\begin{aligned}
\frac{(\mathrm{I}-k) x^{2}}{x} & =\frac{y^{2}+k x^{2}}{y}, x+y=\mathrm{I} ; \\
\therefore \quad(\mathrm{I}-k) x & =2 x^{2}-2 x+\mathrm{I} ; \\
\therefore x & =\frac{1}{4}\left(3-k \pm \sqrt{k^{2}-6} \overline{k+\mathrm{I}}\right) .
\end{aligned}
$$

To take Dr. Wallace's example, put $k=\cdot 1$. Then or

$$
x=\cdot 88508 \ldots, y=\cdot \text { I } 1492 \ldots \text {; }
$$

$$
x=\cdot 56492 \ldots, y=\cdot 43508 \ldots .
$$

Thus, taking Dr. Wallace's number Ioo,, 00 , we find that, ultimately, the normal species will number 88,508 and the variant II,492 These numbers differ but little from Dr. Wallace's, but they represent the final distribution of the original species into twc species. Another possible distribution is given by the numbers 56,492 and 43,508 . If by any chance the first permanent distribution be disturbed materially, then the total species might reach the second permanent state.

If, however, at any time the parent species were to cease to produce the variants, then the latter would quickly disappear. They could be saved from extinction only by the ceasing of intermarriage between the two species. For, if $\left(x_{n}, y_{n}\right)$ denote the $n$th generation from the one $(x, y)$ in which the variants ceased to be produced by the normal species, then

$$
x_{n}: y_{n}:: x^{n}: y^{2 n} \text {. }
$$

If $n=4$, and $x=\cdot 9, y=\cdot 1$, as in Dr. Wallace's case, then

$$
x_{4}: y_{4}::(\cdot 8 \mathrm{I})^{4}: \cdot 0000000 \mathrm{r}
$$

so that the original species of 100,000 would have no variants left at all. The disappearance of the variants is due to the two facts, (I) that the total number of the two species together is constant, (2) that the number of unfruitful unions is very large in proportion to the number of unions possible to the smaller species. For example, if the variants be ' $x$ of the whole species, the probability will be that 9 of their unions will be unfruitful; but that 9 of the unions of the normal species will not be unfruitful.

B. We shall now consider the case when the unions between the two varieties are not sterile, and the hybrids are also fertile inter se and with the parent varieties.

NO. I 544, VOL. 6o]
Let the relative, effective, fertility of the hybrids and mongrels, inter se and with the parent varieties, be denoted by the factor $k$, which we shall assume to be always less than unity. Also, let the effective fertility of the normal species in the production of variants be denoted by the factor $\mu$; and let $z$ denote the number of the hybrid variety in the generation $(x, y, z)$.

Then the equations which determine the stable and permanent condition, if there be one, are

$$
\left.\frac{(\mathrm{I}-\mu) x^{2}}{x}=\frac{y^{2}+\mu x^{2}}{y}=\frac{\left\{(x+y+z)^{2}-x^{2}-y^{9}\right\} \times k}{z}, \ldots \text {. . . I }\right)
$$

Put

then

and

Put
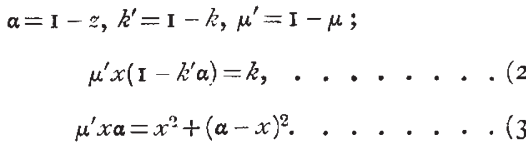

$$
\beta=\left(I-k^{\prime} \alpha\right) \mu^{\prime} \alpha
$$

then

Whence

$$
\beta^{2}-k(3-\mu) \beta+2 k^{2}=0 \text {. }
$$

and

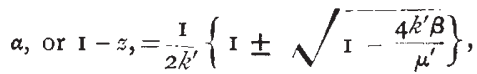

$$
\beta=\frac{k}{2}\left\{3-\mu \pm \sqrt{\mu^{2}-6 \mu+1}\right\} \text {. }
$$

The roots of $\mu^{2}-6 \mu+\mathrm{I}=0$ are $17 \mathrm{I} 58 \ldots$ and $5.82842 \ldots$ As we suppose $\mu$ less than 1 , it follows that in no case must $\mu$ exceed the lower root, 17 I 58 . . . . . . . (8)

From (7) it follows that $\beta$ must $>k$.

From (6) it follows that $\mathrm{I}-\mu$ must $>4 k^{\prime} \beta$, or $=4 k^{\prime} \beta$;

$\therefore \mathrm{I}-\mu$ must $>4 k k^{\prime} ; \therefore k$ must not $>\cdot 5 .$. .

Also $\beta$ must not $>\frac{\mu^{\prime}}{4 k^{\prime}}$

To take Dr. Wallace's example, put $\mu=\cdot I$.

We find, then, that $k k^{\prime}$ must not $>\cdot \mathbf{2 2 5}$. If we put $k k^{\prime}=\cdot 225$, and solve for $k$, we find that

$$
k=342 . . \text {, or } 658 \text {... . }
$$

But $k k^{\prime}$ must not $>\cdot 225$; hence $k$ must not lie between $342 \ldots$ and $658 \ldots$; . by (9) $k$ must not $>342 \ldots$

Take, for example, $k=\cdot \mathbf{2}$.

Then by (7), $\beta=\cdot 225969$. . . or $\cdot 354031$. . .

By (Io) we must reject the second value of $\beta$.

Adopting the first value, we find from equations (2) and (I) the following two solutions,

and

$$
\begin{aligned}
& x=798 . ., y=\cdot 104 . ., z={ }^{\circ} 098 \ldots \\
& x=\cdot 308 \text {. . , } y={ }^{\circ} 040 \ldots ., \ldots=\cdot 652 \ldots
\end{aligned}
$$

Here the effective fertility of the hybrids, inter se and with the parent varieties, must not exceed 34 per cent. of that of the parent varieties; and in no case must it exceed 50 per cent. of the latter. Also, in no case must the parent species supply more than, or even as much as, 18 per cent. of its total progeny to the variant species.

C. If no hybrid unions occur, and the two varieties supply individuals to each other in such a way that, taking the progeny of the generation $(x, y)$, a fraction $\lambda$ of the $x$ progeny belongs to the $y$ variety, while a fraction $\mu$ of the $y$ progeny belongs to the $x$ variety (where $\lambda$ and $\mu$ are proper fractions), it is easy to prove that in the ultimate, established, state of the total species

$$
x: y:: \mu: \lambda \text {. }
$$

Therefore, if $\lambda=\mu$, the species will be, in its final state, equally divided between the two varieties. The equations for the established state are, since now there is no intermarriage,

whence

$$
\frac{x-\lambda x+\mu y}{x}=\frac{y-\mu y+\lambda x}{y}, x+y=\mathbf{I} \text {; }
$$

i.e.

$$
\lambda x=\mu y ;
$$$$
x: y:: \mu: \lambda .
$$

This may also be proved by direct calculation

Woodroffe, Bournemouth.

J. W. Sharpe. 